\title{
BEAM-BASED ALIGNMENT OF TTF RF-GUN USING V-CODE*
}

\author{
W. Beinhauer, R. Cee, W. Koch, M. Krassilnikov ${ }^{\dagger}$, A. Novokhatski ${ }^{\ddagger}$, S. Ratschow, T. Weiland, \\ TEMF, TU-Darmstadt, Germany \\ P. Castro, S. Schreiber, DESY, Hamburg, Germany
}

\begin{abstract}
The beam dynamics simulation code $\mathrm{V}[1,2]$, based on the Ensemble Model [3], is being developed for on-line simulations. One practical application of the V-Code is the beam-based alignment (BBA) of accelerator (TESLA Test Facility) elements. Before we started with BBA the first beam position monitor (BPM1), located after the RFgun cavity, showed non-zero readings. Moreover the readings depended on RF-power, RF-phase and primary and secondary solenoid currents. This effect could be explained by misalignments of the gun and the solenoids. Such beam offsets must be compensated by means of steering coils but such a procedure can be one of the sources of increased emittances. Based on the V-Code solver a dedicated utility was developed for alignment studies. The laser beam mismatch at the cathode, as well as the primary and secondary solenoid displacements were considered as probable reasons for the misalignment of the beam. A new method for the correction of these misalignments combines a sequence of measurements, simulations and the elimination of the largest imperfections. This semi-automatic method applied to the TTF RF-gun yields a centering of the beam within the accuracy of the BPM1.
\end{abstract}

\section{INTRODUCTION}

The TESLA Test Facility (TTF) [4] has been constructed at DESY in order to prove the feasibility and reliability of achieving high accelerating gradients in superconducting RF cavities. The TTF linac [5] can drive a Free Electron Laser (FEL) based on the principle of Self Amplified Spontaneous Emission (SASE) [6]. The SASE FEL requires a high beam quality in terms of low emittances, high peak current and small energy spread. To achieve such performance, we must take care of the mechanical and magnetic alignments to minimize chromatic effects.

The TTF rf gun consists of a $11 / 2$ cell $\mathrm{TM}_{010} \pi$-mode structure operated at $1.3 \mathrm{GHz}$. The sketch of the TTF rf gun is shown in Fig.1. A pair of focusing solenoidal lenses is used to compensate [7] the space charge induced emittance growth. The system of mirrors and irises pro- vides the positioning of the laser at the center of the photocathode. The beam diagnostic is presented by a button type Beam Position Monitor (BPM1).

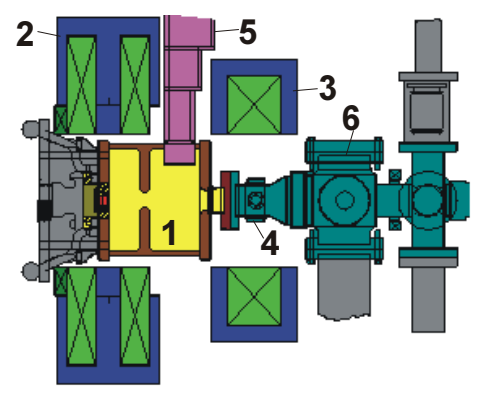

Figure 1: TTF rf gun: 1 - rf cavity; 2 - primary solenoid; 3 - secondary solenoid; 4 - beam position monitor

(BPM1); 5 - rf coupling waveguide; 6 - laser port.

It has been observed that the BPM1, located after the RF-gun cavity, showed non-zero readings. Moreover it was found that the readings depend on rf-power, rf-phase and primary and secondary solenoid currents. The dependencies of the beam position $\left(\mathrm{X}_{\mathrm{BPM} 1}, \mathrm{Y}_{\mathrm{BPM} 1}\right)$ on solenoid currents are shown in Fig.2. All these indicate the existence of misalignments between the components of the rf gun.

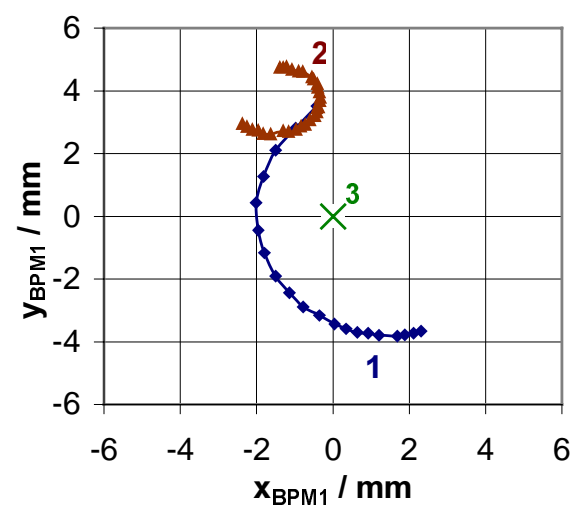

Figure 2: Measured beam position $\left(\mathrm{X}_{\mathrm{BPM} 1}, \mathrm{Y}_{\mathrm{BPM} 1}\right)$ dependence on: 1 - primary solenoid current

$\left(0 \mathrm{~A}<\mathrm{I}_{\text {sol } 1}<400 \mathrm{~A}, \mathrm{I}_{\text {sol } 2}=0 \mathrm{~A}\right) ; 2-$ secondary solenoid current $\left(-290 \mathrm{~A}<\mathrm{I}_{\text {sol } 2}<290 \mathrm{~A}, \mathrm{I}_{\mathrm{sol} 1}=0 \mathrm{~A}\right) .3-\mathrm{BPM} 1$ center.

\footnotetext{
* Work supported in part by DESY, Germany and DFG (FOR 272/2-1).

${ }^{\dagger}$ kras@temf.de

$¥$ Now at SLAC
} 
In the case of a perfectly aligned gun the beam positions do not depend on solenoid currents and must coincide with the BPM center. The nonzero readings could be explained by misalignments of the gun and the solenoids. Even small construction errors cause a difference in the positions of the mechanical and magnetic solenoid centers, producing a steering force acting on the bunch at the cavity axis. The laser beam offset at the cathode also can explain part of the beam deflection. These misalignments can not be measured directly and thus the Beam Based Alignment (BBA) has been applied.

\section{ALIGNMENT CONCEPT}

\subsection{Analytical Model}

Qualitative analytical estimations can be done in order to explain the electron beam position dependencies (Fig.2). The magnetic field of the primary $H_{z}^{(1)}$ and secondary $H_{z}^{(2)}$ solenoid can be approximated with high accuracy (Fig.3a) by formulae for coils with iron shield [8]:

$$
\begin{aligned}
& H_{z}^{(1)}(z)=H_{0}^{(1)} \cdot\left[F\left(z, z_{1}, a_{1}\right)-F\left(z,-z_{1}, a_{1}\right)\right], \\
& H_{z}^{(2)}(z)=H_{0}^{(2)} \cdot F\left(z, z_{2}, a_{2}\right),
\end{aligned}
$$

where $F\left(z, z_{c}, a\right)=\left[\cosh \left(\frac{z-z_{c}}{0.53 \cdot a}\right)\right]^{-1}, z_{1,2}$ are coil center positions, $a_{1,2}$ - solenoids apertures, $H_{0}^{(1,2)}$ are solenoid currents functions.
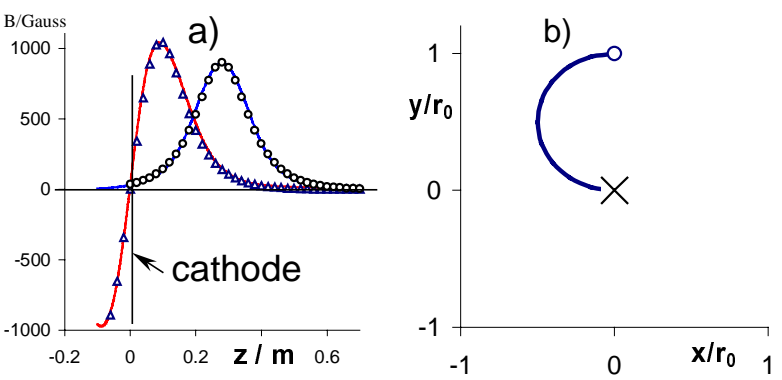

Figure 3: a) Magnetic field of the solenoids (solid lines) and their approximation (1) for primary (triangle markers) and secondary coil (circles). b) Beam position dependence on solenoid peak field. The circle denotes the initial beam offset, the cross is the solenoid center.

Using the expressions (1) for the solenoidal field one obtains from Busch's theorem [8,9] the formulae for the beam position (radius-vector $r$ and angle $\theta$ ):

$$
\frac{r}{r_{0}} \approx \cos \left(\alpha_{1,2} H_{0}^{(1,2)}\right), \quad \Delta \theta \approx-\pi \alpha_{1,2} H_{0}^{(1,2)},
$$

where parameter $\alpha_{1,2}=\frac{e a_{1,2}}{m c^{2} \sqrt{\gamma^{2}-1}}$ and $r_{0}$ is initial beam offset from the solenoid center. The typical beam position dependence on solenoid current for the vertical initial beam offset is shown in Fig. 3 b.

The formulae (2) do not take into account beam acceleration by the rf field, as well as focusing effect from the rf field, but it qualitatively correctly describes a beam missteering due to solenoid misalignment and helps to find a preliminary position of the solenoid magnetic center and laser offset at the cathode.

\subsection{V-Code Alignment Utility}

$\mathrm{V}$-code based on the Ensemble Model [3] is a powerful tool for the beam dynamics simulations for entire accelerator, including low beam energy areas [1]. The beam line data base of the code is designed in order to be compatible with the control system, which provides the machine parameters on-line acquisition [2]. The Alignment Utility, derived from the V-Code solver was applied to the TTF rf gun alignment. The misalignments correction algorithm consists of:

I. Measurements of dependencies of beam position on solenoid current (with the other solenoid switched off) with saving all machine parameters.

II. Simulations with saved machine parameters in order to obtain solenoids magnetic centers and laser beam offset at the cathode.

III. Elimination of the largest imperfections with corresponding screws tuning.

In the next section 3 , it is demonstrated that a proper iteration of I-III yields a better beam centering.

The misalignment search algorithm, implemented in the Alignment Utility, involves the measurement errors. The analytical model helps to find a proper misalignment range and significantly accelerates the search.

\section{RF-GUN ALIGNMENT}

The BPM1 readings presented in Fig. 2 were taken as initial measurements for the BBA. First misalignments simulations yielded the results shown in Fig.4.

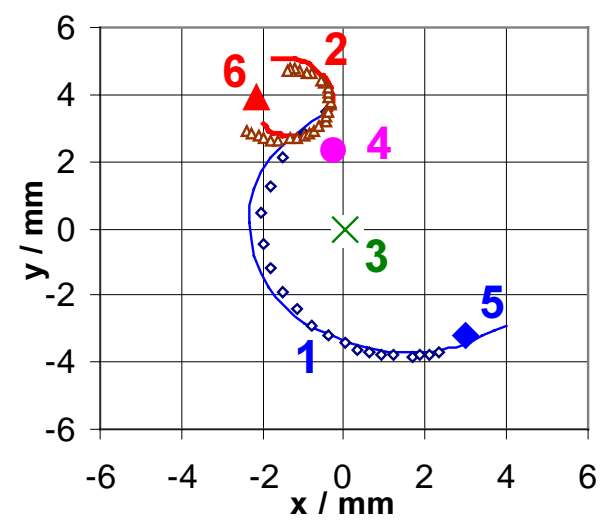

Figure 4: Initial simulation results. Beam position dependence on solenoids currents: measured (markers) and simulated (solid lines): 1- primary solenoid. 2 secondary solenoid. 3 - BPM center. 4 - simulated laser position at the cathode. 5 - simulated primary solenoid center. 6 - simulated secondary solenoid center. 
It should be noted that simulated magnetic centers of the solenoids are in rather good agreement with predictions of the analytical model (2). The beam start point (laser position at the cathode and the solenoid magnetic center are located along the diameter of the corresponding arc (see Fig.2b and Fig.4).

The vertical alignment of the secondary solenoid was suggested as a first alignment manipulation. The secondary solenoid was moved down by $3 \mathrm{~mm}$. The new position of the magnetic center is shown in Fig. 5 by triangle marker 6a. Solid curve 2a corresponds to the simulations predictions. The measured points after the solenoid alignment are denoted by filled triangles. The discrepancy can be explained by model of the solenoids magnetic field implemented in the V-code. The beam line element TTF$R F$-Gun in the V-code data base contains two independent solenoids (and a small trim coil for the fine compensation of the magnetic field at the cathode). The fields are scaled by actual solenoid currents, nonlinear saturation is implemented for the primary solenoid, the secondary coil is scaled linearly by the current. The present version of the code does not take into account a mutual influence of the solenoids and hysteresis effects. Although the position of the primary solenoid was not changed during the first alignment step, the beam positions shifted up, when the dependence on the solenoid 1 current was measured once more (filled rhombic markers in Fig.5). This can be explained by fields, induced by primary coil in the secondary solenoid yoke (which vertical position was changed).

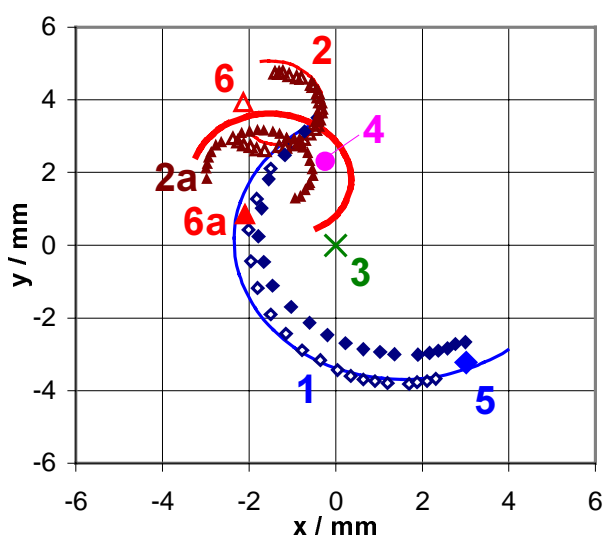

Figure 5: Results after first iteration, where secondary solenoid (6) was moved down by $3 \mathrm{~mm}$.

(For denotations see Fig.4 caption.)

The new measurements were taken as a calculation pattern for the next iteration in the alignment procedure. The final configuration and corresponding beam position dependencies after several steps of the alignment are shown in Fig.6. Both solenoids centers, laser position at the cathode and BPM center are within a box $2 \mathrm{~mm} \times 2 \mathrm{~mm}$ During alignment procedure horizontal and vertical movement of the solenoids and laser beam correction were involved.

The beam offset for the operating machine parameters was reduced significantly (Tab.1).

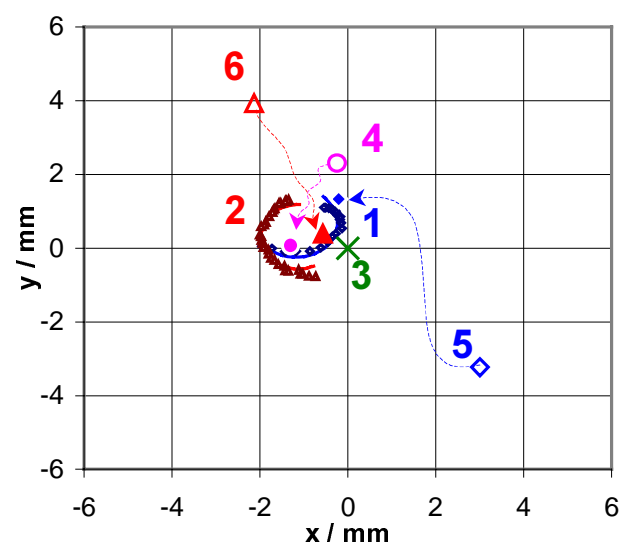

Figure 6: Results after beam-based alignment. Movement of solenoids centers and laser position is shown by arrows. (For denotations see Fig.4 caption.)

Table 1: BPM1 readings for nominal settings of solenoids (only statistical errors included).

\begin{tabular}{|l|c|c|}
\hline & Before BBA & After BBA \\
\hline $\mathrm{X}_{\mathrm{BMP}} / \mathrm{mm}$ & $4.72 \pm 0.16$ & $0.42 \pm 0.06$ \\
\hline $\mathrm{Y}_{\mathrm{BMP}} / \mathrm{mm}$ & $-1.35 \pm 0.07$ & $0.80 \pm 0.05$ \\
\hline
\end{tabular}

\section{CONCLUSION}

A new method for the correction of accelerator devices misalignments based on the V-Code is proposed. It has been shown that the gun solenoids and laser position at the cathode can be aligned using beam based alignment with V-code utility. This semi-automatic procedure applied to the TTF RF-gun yields a centering of the beam within the accuracy of the BPM.

\section{REFERENCES}

[1] A.Novokhatski, T.Weiland, M.Krassilnikov, W.Koch, P.Castro, "Beam Dynamics On-Line Simulation", LINAC 2000, Monterey, Proc. pp.836-838.

[2] M.Krassilnkov, A.Novokhatski, T.Weiland, W.Koch, P.Castro "V-Code On-Line Simulation of Collective Beam Effects" ICAP2000. http://www.icap2000.de/

[3] A.Novokhatski, T.Weiland, "The Model of Ensemble for the Beam Dynamics Simulation", Proc. of PAC99, p. 2743

[4] TESLA Technical Design Report, DESY 2001-011.

[5] TESLA Test Facility. Design Report. DESY Print. March 1995, TESLA 95-01.

[6] J.Andruszkow et al., "First Observation of SelfAmplified Spontaneous Emission in a Free-Electron Laser at $109 \mathrm{~nm}$ Wavelength", Phys. Rev. Lett. 85 (2000) 3825.

[7] B.E. Carlsten et al., Nucl. Instr. And Meth. A285 (1989) 313.

[8] I.N.Meshkov, "Transport of the charged particles", Nauka, Novosibirsk, 1991.

[9] M.Reiser, "Theory and Design of Charged Particles Beams", John Wiley \& Sons, Inc., 1994. 